\title{
ON SHAPE FROM SHADING AND SAR IMAGES: AN OVERVIEW AND A NEW PERSPECTIVE
}

\author{
Gerardo Di Martino, Alessio Di Simone, Antonio Iodice, Daniele Riccio, Giuseppe Ruello
}

\author{
Università di Napoli Federico II, Via Claudio 21, 80125, Napoli, Italy
}

\begin{abstract}
In this paper, we first discuss some general problems and issues concerning Shape from Shading (SfS) from Synthetic Aperture Radar (SAR) images and present the state of the art. Starting from this general discussion, we propose a new perspective and introduce a novel approach to SAR SfS based on fractals. In particular, fractal geometry is used to model the shape of natural surfaces, which are here of concern. The scattering mechanisms are described through solutions suitable for fractal surface models; in particular, the Small Perturbation Method (SPM) is used. Considering a simple SAR image model, the forward model is then inverted via an appropriate and extremely lowcomputational inversion approach and the underlying topography is estimated. The proposed SAR SfS technique is tested and numerically evaluated using an actual SAR image.
\end{abstract}

Index Terms - Synthetic Aperture Radar, shape from shading, fractals, digital elevation model

\section{INTRODUCTION}

The problem of Shape from Shading is to determine the shape of a surface given a single gray-level image of that surface. The image can be acquired in the optical spectrum (photo) or in the microwave region (radar or SAR image). In other words, SfS is one of the existing techniques to recover the shape of an object from the reflectivity intensity map of the corresponding surface. The main prerequisite to achieve this goal is the knowledge of the reflectivity function of the surface in the spectral region of interest.

The study of shape from shading goes back to the development of lunar topography from lunar imagery by J. van Diggelen in 1951 [1]. He considered the possibility of using optical images of the moon (i.e., the brightness of the moon's surface) to recover the moon's topography; in particular he focused on the well-known Mare Imbrium, a vast lunar mare filling a basin, which was created when lava flooded the giant crater formed when a very large object hit the Moon long time ago. As stated by van Diggelen himself, until then the topography of the Moon was recovered estimating the lengths of the shadows using a purely geometric method, which he showed to be unsuitable for the large portion of the surface of the Moon formed by the smooth landscape of the Maria. Therefore, he was the first to propose the cosine (Lambertian) law for the brightness function.

Since the seminal work by J. van Diggelen [1], and the subsequent works of Wildey [4]-[5], Horn [6]-[7], Frankot and Chellappa [8], SfS techniques have now reached a high level of accuracy, reliability and robustness within the area of optical imaging, where very accurate SfS algorithms have been developed. Conversely, very little has been obtained from SfS applied to SAR images. Actually, the extension to SAR is not a trivial issue, because of the specific geometric and electromagnetic (scattering) characteristics of microwave SAR systems. Thus, in the last decade, due also to the increasing availability of SAR interferometric products, the interest in SAR shape from shading has been decreasing. However, in some cases interferometric SAR configurations cannot be attained: the most obvious example is in the context of extraterrestrial missions, where even a rough knowledge of the topography (in terms of local slopes, rather than of absolute height) can greatly help in the interpretation of geodynamical processes.

In the next section we briefly discuss the limits and the state of the art of SAR SfS techniques, while in Section 3 we outline some new perspectives and propose a new SfS technique based on fractals [3], [10].

\section{SHAPE FROM SHADING AND SAR IMAGES}

Up to now, many techniques aimed at retrieving the surface elevation profile have been developed, namely stereoscopy [18], interferometry [19], radarclinometry (or shape-fromshading) [4]-[9] and polarimetry [13] each with its own advantages and drawbacks. Unlike the other techniques, which require (a minimum of) two complex SAR images possibly acquired at the same time, shape-from-shading has the main feature and advantage of requiring only one intensity image. In principle, the former techniques rely on simple principles, but suffer from a series of problems related to decorrelation and availability of multiple coherent acquisitions. Conversely, SfS is in principle unfeasible without entering the scattering issues, but do not prescribe any particular acquisition strategy. This makes SfS conventionally suitable (if the case) if a (very) low approximate DEM is required and a single (generic) SAR 
image is only available. However, many problems arise with any SfS method: this is due to the huge number of parameters (inherent to both the sensor and the surface, like frequency, radar look angle, resolution, chirp bandwidth, macroscopic and microscopic roughness, local slopes, complex dielectric constant) influencing the surface scattering and then SAR image formation. As a result, SfS is an ill-posed problem: a unique equation dependent on a large number of unknown parameters, of which only local slopes are of interest. The nonlinear contribution of these parameters to the scattering mechanism makes these dependences so involved that only approximate solutions can be derived; in addition, geometrical distortions and speckle cause detrimental effects on the performance of SAR SfS technique and have to be properly modeled and faced. Layover and shadowing cause severe distortions in the SAR image and then could irreversibly affect the technique, but fortunately they are present only in presence of sufficiently high slopes; on the contrary, foreshortening is always present and causes distortions that increase with the local slopes of the surface, and consequently its effects are negligible in case of sufficiently small slopes.

Since the underlying topography is estimated directly from intensity data, SfS shows a great sensitivity to all the phenomena that influence the received intensity, firstly speckle. Since speckle is signal dependent, its influence on dark reverse slopes and bright forward slopes will be different. In SAR SfS speckle results in DEM random oscillations also in presence of a constant height surface, assuming all other parameters influencing the algorithm spatially constant.

\section{A NEW PERSPECTIVE FOR SAR SFS}

Besides the aforementioned reasons and difficulties, the main reason for the discrepancy between the performances of optical and SAR SfS techniques lays in the widespread use of the so-called Lambertian scattering model [9], [13], [14] (or its generalization [9], [12]), which, although valid in optics for scattering from matte surfaces, is very inaccurate in describing scattering from natural surfaces in the microwave region of the spectrum, where SAR sensors usually operate. Actually, Lambertian scattering prescribes that the observed surface scatters the radar waves equally in all directions and that the image brightness is proportional to the cosine of the local incidence angle. Conversely, theoretical and experimental studies [9]-[11], [14], [15], proved that at microwaves the scattering phenomenon is also influenced, among other factors, by the type of terrain (in terms of its electromagnetic characteristics), the level of humidity, the wavelength, the polarization. Indeed, there is increasing experimental and theoretical evidence that the fractal geometry represents the most appropriate mathematical environment to describe the shape of natural surfaces [2], [3], [12], [16], [17]; furthermore, this fractal approach provides a description of the surface with a minimum number of independent parameters [10]. However, it is not immediate to transfer known conventional scattering computational techniques to the fractal geometry: a complete rephrasing is necessary. An accurate and complete study about scattering from fractals surfaces is developed in [10].

In this paper, we propose a new technique for SAR SfS based on the use of fractal models. In particular, the paper is aimed at showing that strengthening the direct model introducing proper models both for surface and scattering mechanisms, it is possible to estimate with sufficient accuracy the underlying topography also with a very simple and low-computational-complexity inversion technique. To this aim, the proposed direct model is divided in three parts:

\section{- $\quad$ Surface model}

The natural surface under study is properly modeled using fractal geometry; in particular, a fractional Brownian motion $(\mathrm{fBm})$ is used [6]:

$\operatorname{Pr}\left\{z(x, y)-z\left(x^{\prime}, y^{\prime}\right)<\bar{\zeta}\right\}=\frac{1}{\sqrt{2 \pi} s \tau^{H}} \int_{-\infty}^{\bar{\zeta}} \exp \left(-\frac{\zeta^{2}}{2 s^{2} \tau^{2 H}}\right) d \zeta$

where Pr stands for "probability", $\tau$ is the distance between the two considered points of coordinates $(x, y)$ and $\left(x^{\prime}, y^{\prime}\right)$; $H$ is the Hurst coefficient $(0<H<1)$; $s$ is the incremental standard deviation.

- $\quad$ Scattering model

In general, only approximate analytic solutions to the scattered field are achievable for natural surfaces. In this work, the scattering mechanisms are described through the SPM for fBm surfaces because of its simplicity and range of validity adequate to SAR applications [7]:

$$
\sigma_{m n}^{0}=2 \pi 8 k^{4} \cos ^{4} \theta\left|\beta_{m n}\right|^{2} \frac{S_{0}}{(2 k \sin \vartheta)^{2+2 H}}
$$

wherein $k$ is the electromagnetic wavenumber of the incident field; $S_{0}$ is the spectral amplitude of the $\mathrm{fBm}$ surface; $\beta_{m n}$ is a coefficient depending on transmitted and received signal polarization and the radar look angle $\theta$.

- Imaging model

The intensity of a SAR image can be assumed as the amount of electromagnetic energy backscattered from the resolution cell. Assuming a small-slopes regime for the surface a firstorder approximation of the image intensity can be evaluated with regard to the local range slopes of the observed surface $p$ (in a first order approximation no dependence on the azimuth slopes is present [8]):

$$
I=G\left(a_{0}+a_{1} p\right)
$$


TABLE I

PERFORMANCE INDICATORS FOR THE FRACTAL AND LAMBERTIAN MODEL FOR THE SAR IMAGE OF THE VESUVIUS VOLCANO

\begin{tabular}{|c|c|c|c|c|c|c|c|c|c|c|c|}
\hline \multirow{2}{*}{\multicolumn{3}{|c|}{ Error magnitude }} & \multicolumn{3}{|c|}{ Altitude $(\mathrm{m})$} & \multicolumn{3}{|c|}{ Range slope $\left({ }^{\circ}\right)$} & \multicolumn{3}{|c|}{ Azimuth slope $\left(^{\circ}\right)$} \\
\hline & & & Median & Mean & Std dev. & Median & Mean & Std dev. & Median & Mean & Std dev. \\
\hline \multirow{2}{*}{\multicolumn{2}{|c|}{$\begin{array}{l}\text { Before azimuth } \\
\text { filtering }\end{array}$}} & Fractal Model & 142.5 & 166.8 & 120.8 & 9.62 & 11.95 & 10.73 & 21.57 & 27.39 & 22.27 \\
\hline & & Lambertian Model & 267.2 & 413.6 & 446.2 & 25.58 & 26.39 & 17.99 & 69.50 & 65.51 & 28.34 \\
\hline \multirow{4}{*}{$\begin{array}{l}\text { After } \\
\text { azimuth } \\
\text { filtering }\end{array}$} & \multirow{2}{*}{$\begin{array}{l}\text { Unknown } \\
\text { starting } \\
\text { points }\end{array}$} & Fractal Model & 142.4 & 166.8 & 120.7 & 9.60 & 11.94 & 10.63 & 12.21 & 15.30 & 13.16 \\
\hline & & Lambertian Model & 208.5 & 364.0 & 444.2 & 21.15 & 26.01 & 21.91 & 36.18 & 39.68 & 27.06 \\
\hline & \multirow{2}{*}{$\begin{array}{l}\text { Known } \\
\text { starting } \\
\text { points }\end{array}$} & Fractal Model & 98.7 & 126.3 & 105.3 & 9.60 & 11.94 & 10.63 & 9.67 & 14.14 & 15.20 \\
\hline & & Lambertian Model & 155.8 & 321.1 & 454.5 & 21.15 & 26.01 & 21.91 & 33.98 & 37.87 & 26.73 \\
\hline
\end{tabular}

The proposed inversion technique allows retrieving an estimate of the slopes of the surface in the range direction, which can be integrated to obtain an estimate of the corresponding elevation profile. Assuming that the mean value of the intensity map $(<I>)$ is a good estimation of the intensity linked to a flat surface, we calculate $G$ as follows

$$
G=\frac{<I>}{a_{0}} .
$$

To take into account the azimuth slopes (which are usually neglected in other techniques [4]-[9] or introduced using polarimetric concepts [13]) we propose the use of a regularization procedure based on Bayesian Minimum Mean Squared Error (MMSE) estimation.

The result obtained applying the proposed technique on a multilook Cosmo/SkyMed stripmap SAR image of the Somma-Vesuvius volcanic complex, close to Naples, Italy, are presented in Fig. 1 and Fig. 2. In particular in Fig. 1 (c)(d) the estimated DEMs before and after the azimuth regularization procedure are shown assuming unknown starting points. As can be seen, the azimuth filtering greatly reduces the linage effects clearly visible in Fig. 1 (c). In Fig. 2 range and azimuth profiles of the estimated DEM are reported and compared with those obtained using a Lambertian model. A quantitative assessment of the proposed method is reported in Table I, where the benefits provided by the fractal model can be clearly appreciated.

\section{AKNOWLEDGEMENT}

This work was partly funded by ASI in the framework of the AO project "Exploitation of fractal scattering models for Cosmo/SkyMed images interpretation”.

\section{REFERENCES}

[1] J. van Diggelen, "A photometric investigation of the slopes and the heights of the ranges of hills in the Maria of the moon," Bull. Astron. Inst. Netherlands, vol. 11, no. 243, pp. 283-290, 1951.

[2] W. W. Hargrove, F. M. Hoffman, and P. M. Schwartz, "A fractal landscape realizer for generating synthetic maps," Conservation Ecology, vol. 6, no. 1, Oct. 2002.

[3] B. Mandelbrot, The Fractal Geometry of Nature. New York: Freeman, 1983.
[4] R. L. Wildey, "Radarclinometry for the Venus radar mapper," Photogramm. Eng. Remote Sensing, vol. 52, no. 1, pp.41-50, 1986. [5] R. L. Wildey, "The surface integral approach to radarclinometry," Earth, Moon, Planets, vol. 41, pp. 141-153, 1988.

[6] B. K. P. Horn, "Obtaining shapes from shading information," in Psychology of Computer Vision. New York: McGraw-Hill, pp. 115-155, 1975.

[7] B. K. P. Horn and M. J. Brooks, Eds., Shape From Shading. Cambridge, MA: MIT Press, 1989.

[8] R. T. Frankot and R. Chellappa, "A method for enforcing integrability in shape from shading algorithms," IEEE Trans. Pattern Anal. Machine Intell., vol. 10, pp. 439-451, Mar. 1988.

[9] D. N. Ostrov, "Boundary Conditions and Fast Algorithms for Surface Reconstructions from Synthetic Aperture Radar Data," IEEE Trans. Geosci. Remote Sens., vol. 37, no. 1, pp. 335-346, Jan. 1999.

[10] G. Franceschetti and D. Riccio, Scattering, Natural Surfaces and Fractals. Burlington, MA: Academic, 2007.

[11] G. Ruello, P. Blanco, A. Iodice, J.J. Mallorqui, D. Riccio, A. Broquetas and G. Franceschetti, "Synthesis, construction and validation of a fractal surface," IEEE Trans. Geosci. Remote Sens., vol. 44, no. 6, pp. 1403-1412, Jun. 2006.

[12] M. Oren, S. K. Nayar, "Generalization of Lambert's Reflectance Model," in Proceedings of the 21st annual conference on Computer graphics and interactive techniques, pp. 239-246, 1994.

[13] X. Chen, C. Wang, H. Zhang, "DEM Generation Combining SAR Polarimetry and Shape-From-Shading Techniques," IEEE Geoscience and Remote Sensing Letters, vol. 6, no. 1, pp. 28-32, Jan. 2009.

[14] R. T. Frankot and R. Chellappa, "Estimation of Surface Topography from SAR Imagery Using Shape from Shading Techniques," Artificial Intelligence, vol. 43, pp. 271-310, 1990.

[15] P. Fua, "Fast, Accurate and Consistent Modeling of Drainage and Surrounding Terrain," Int. J. Computer Vision, vol. 26, no. 3, pp. 215-234, 1998.

[16] W. G. Carrara, R. S. Goodman, and R. M. Majewski, Spotlight Synthetic Aperture Radar Signal Processing Algorithms. Artech House, 1995. (b)

[17] M. I. Skolnik, Radar Handbook. New York: McGraw-Hill, 1970.

[18] Dhond, U.R. and Aggarwal J.K., "Structure from stereo - a review," IEEE Transactions on Systems, Man and Cybernetics, Vol. 19, No. 6, pages 1489-1510, Nov. 1989.

[19] P. A. Rosen, S. Hensley, I. R. Joughin, F. K. Li, S. N. Madsen, E. Rodríguez, and R. M. Goldstein, "Synthetic Aperture Radar Interferometry," Proc. IEEE, vol. 88, no. 3, pp. 333-382, March 2000. 


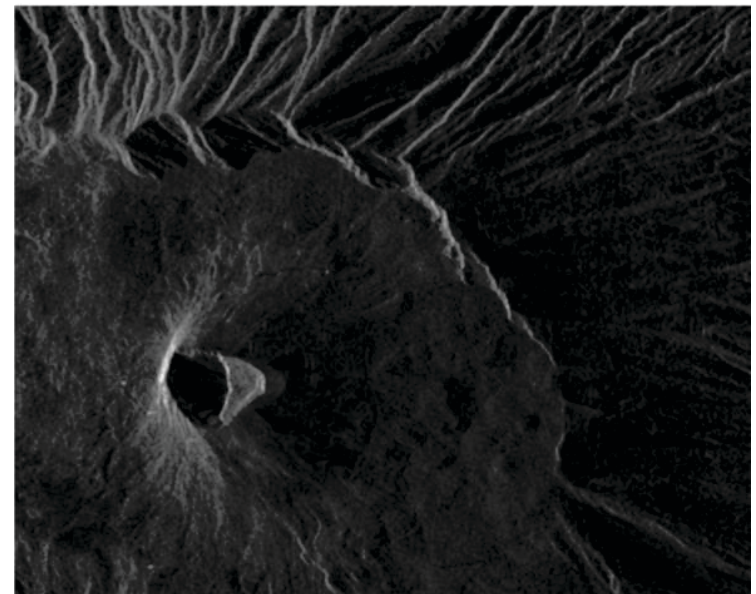

(a)

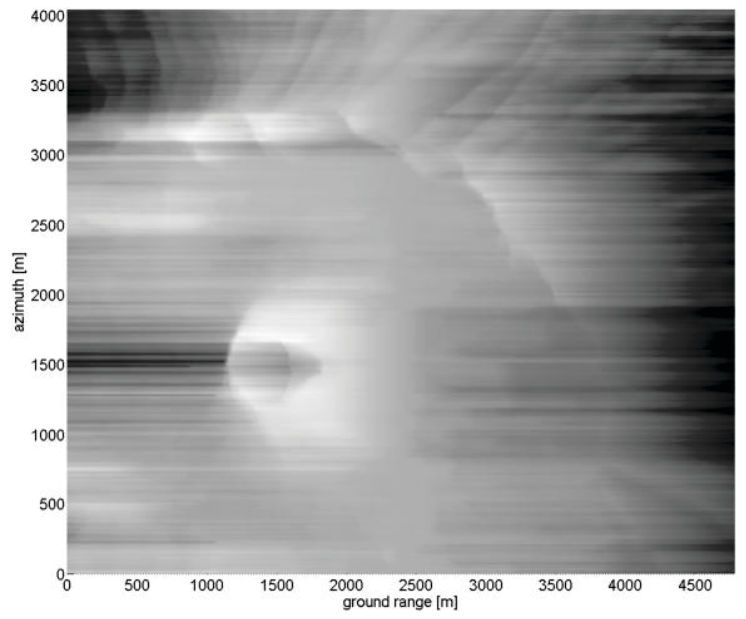

(c)

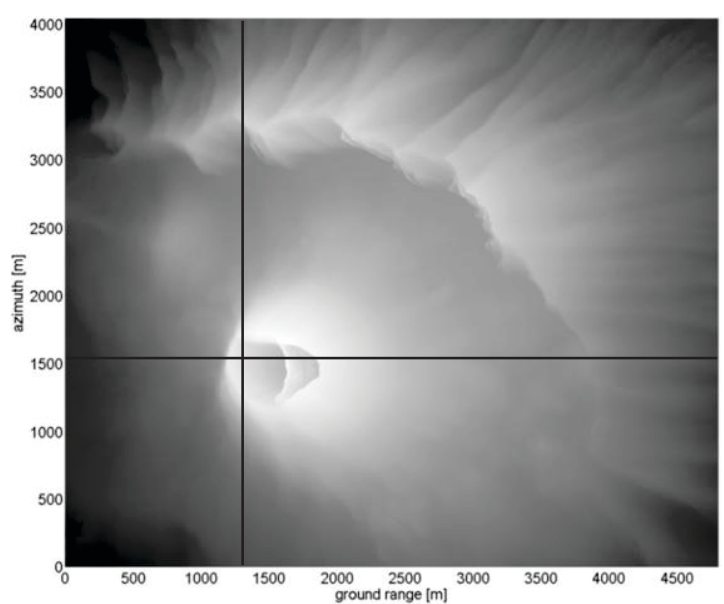

(b)

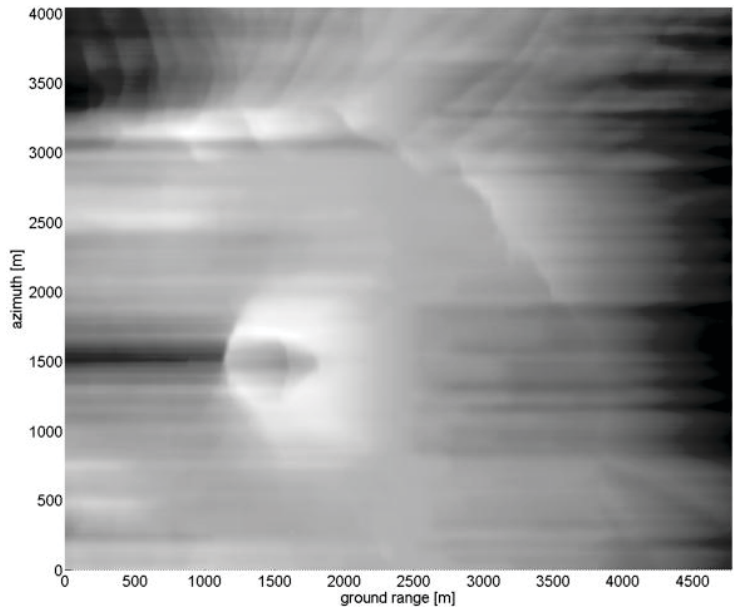

(d)

Fig. 1 Cosmo/SkyMed SAR image of the Vesuvius complex (a); ground-truth DEM in azimuth-slant range coordinates (b) with lines identifying range and azimuth cuts; obtained SfS DEM before (c) and after (d) azimuth regularization procedure assuming unknown starting points in the range integration step.
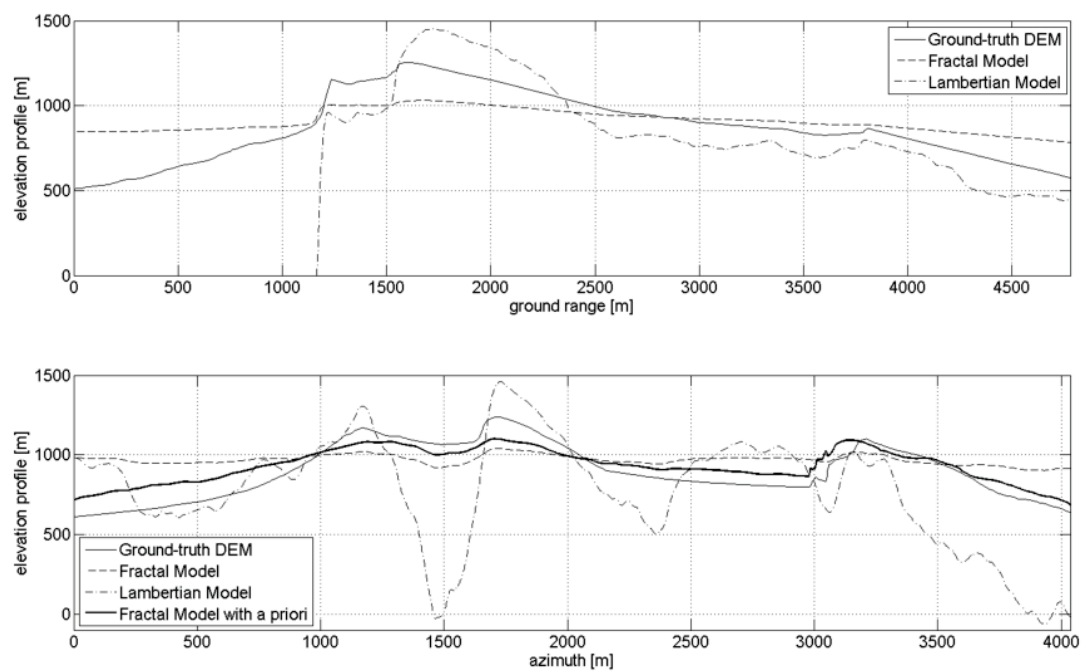

Fig. 2 Range (top) and azimuth (bottom) cuts of the obtained SfS DEM. Better results provided by the fractal model are visible especially in the azimuth cut, where a priori knowledge about starting points in the range integration step can improve performances. 